Thorax (1958), 13, 218.

\title{
DESMOID TUMOUR OF THE CHEST
}

\author{
BY \\ WENDELL K. NICKELL, C. FREDERICK KITTLE, AND JAMES O. BOLEY \\ From the Departments of Surgery and Pathology, University of Kansas Medical Center, \\ Kansas City, Kansas
}

(RECEIVED FOR PUBLICATION APRIL 8, 1958)

Desmoid tumours are uncommon fibrous neaplasms usually originating from fascia of the anterior rectus muscle in multiparous women. Extra-abdominal desmoids are rare and present certain problems in their diagnosis and operative management. We should like to describe a patient with a desmoid tumour of the chest wall recently treated at the University of Kansas Medical Center.

\section{CASE History}

B. H., a 21-year-old housewife, was first seen on February 27, 1956, complaining of a gradually enlarging painless nodule in the left chest wall of six months' duration. No history of trauma, inflammation, or rapid growth could be elicited. The patient had experienced a normal pregnancy and delivery two years previously. When first seen she had had amenorrhoea and morning nausea for two months. Otherwise the history contributed nothing.

Physical examination revealed a healthy adult woman with evidence of early intra-uterine pregnancy. Abnormal findings were confined to the chest. Immediately adjacent to the left sternal border was a body-hard, immovable mass about $3 \times 4 \mathrm{~cm}$. in diameter and protruding $1.5 \mathrm{~cm}$. above the surrounding tissues. It extended from the third to the fourth intercostal spaces and obscured the fourth costal cartilage. The skin was not involved.

Because of the consistency of the mass and its relation to the costal cartilage, a pre-operative diagnosis of chondroma, chondrosarcoma, or fibrosarcoma seemed most likely.

Routine laboratory studies as well as serum alkaline phosphatase were normal. Radiographs of the chest with particular views for rib detail revealed no abnormality. Surgical excision was advised.

On March 10, 1956, the mass was approached through a left inframammary incision extending across the sternum at the level of the third interspace. The tumour mass occupied the third and fourth interspaces in the region of the costal cartilages, but did not involve the subcutaneous tissue.

An incision was made through the fourth interspace for intrapleural palpation. No evidence of pleural invasion was found. The mass and contiguous chest wall was excised en bloc. This included the third and $\vec{\omega}$ fourth costal cartilages, the soft tissues from the $\omega$ second to the fourth interspaces, the overlying pectoral muscle, and a portion of the sternum (Fig. 1). Stability of the chest in the area of resection was established by suturing fibreglass mesh to the edges of the defect. Coverage was easily accomplished with $\mathcal{C}$ the left breast after instituting intrapleural drainage.

Post-operatively the patient's course was uneventful and she was discharged on the ninth post-operative day. When last seen in January, 1958, approximately 22 months post-operatively, there was no evidence by either physical or radiological examination of recurrence.

Pathological Description. - Gross inspectio̊ showed a firm elastic, $5 \times 4 \times 2 \mathrm{~cm}$. unencapsulate mass which replaced intercostal tissue, although îे had not invaded the cartilage, sternum, or pleur The tissue was pinkish-white and appeared macroscopically to consist of bundles of fibrous tissue with a whorled pattern (Fig. 2). Histological examination revealed masses and strands of well-differentiated, moderately cellular, fibrous tissue invading and replacing adjacent striated muscle (Figs. 3 and 4). Occasional mitoses were noted. No tissue other than muscle was invaded and excision was believed to be complete. The diagnosis was desmoid tumour of the anterior chest wall.

\section{Discussion}

First recognized grossly by MacFarlane in 1832 and described microscopically by Bennett in 1850, 욱 the term "desmoid" was introduced by Müller in $D$ 1838 to designate a fibrous, benign tumour of tendinous consistency. Its predominance in multi- $\vec{N}$ parous women and its aetiological relation to the $\Omega$ trauma of pregnancy, previous operations, and $\tilde{O}$ local injury have been generally emphasized. A few instances of congenital desmoid tumours have 으 been reported (Greig, 1915).

Initially the term desmoid was restricted to a $\frac{0}{D}$ tumour arising from the aponeurosis of the abdo- $\stackrel{\mathcal{D}}{?}$ minal wall musculature (Stewart and Mouat, 0 1924). Subsequently extra-abdominal tumours histologically resembling desmoids have been de- 


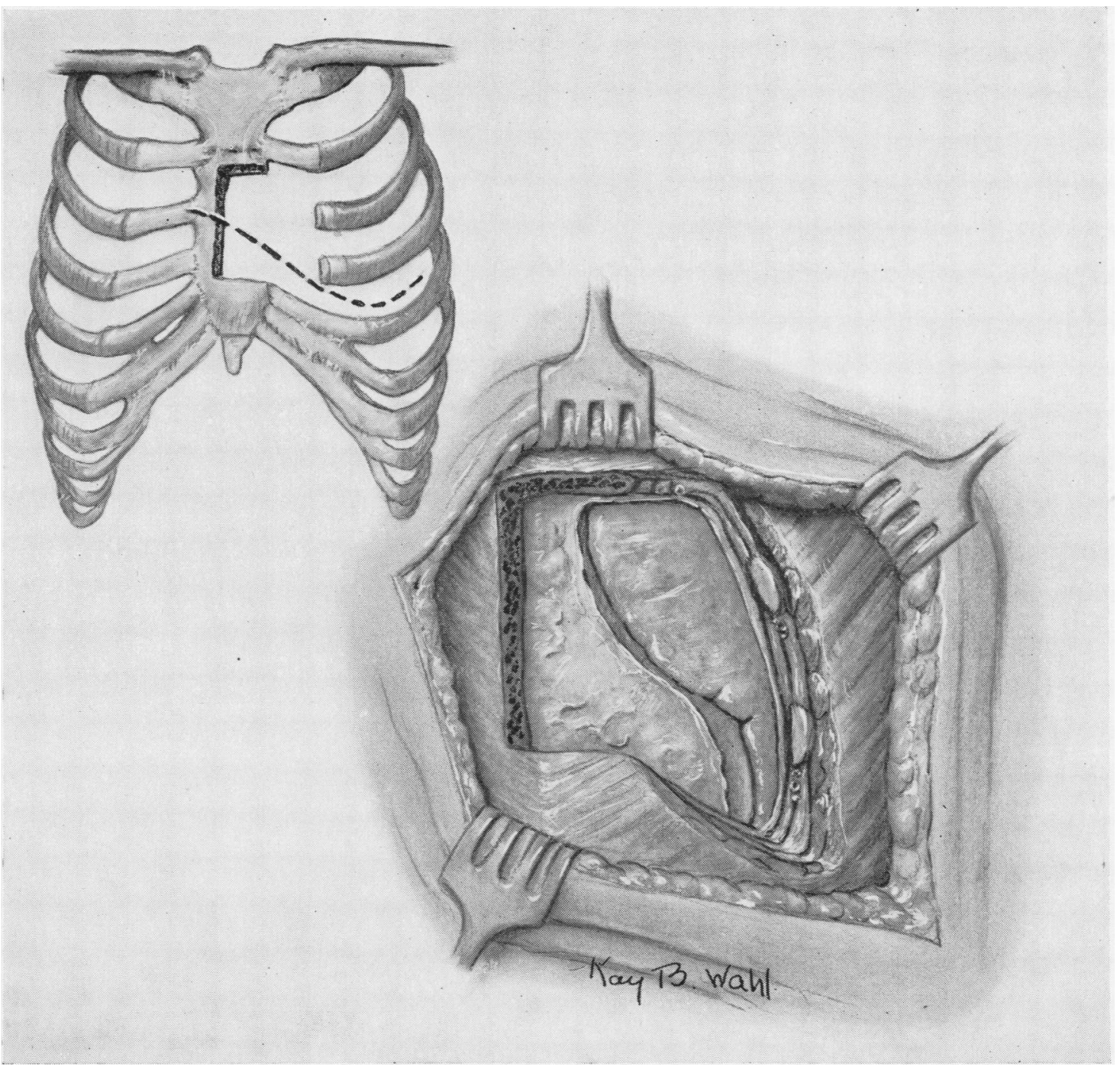

FIG. 1.-Sketch of operative excision.

scribed (Nichols, 1923 ; Mason, 1930 ; Waugh, 1940 ; Pearman and Mayo, 1942). The definition has been modified to include any fibroplastic tumour of benign nature that is unencapsulated and invades adjacent striated muscle.

Desmoid tumours occur infrequently. Musgrove and McDonald (1948) reported 130 cases seen at the Mayo Clinic over a 40-year period. Pack and Ehrlich (1944) described 17 abdominal desmoids seen at Memorial Hospital from 1917 to 1943 among 50,340 cases of neoplasm. In 82,532 admissions to the University of Kansas Medical Center from 1952 to the end of 1957, there have been four patients with desmoid tumours, three abdominal and one extraabdominal, the last being the present case.

Desmoids originating within the chest wall are rare. Gatchell, Clagett, and McDonald (1957) presented an intercostal desmoid and cited it as the first published case. However, Musgrove and McDonald (1948) had described a desmoid of the intercostal muscles following radical mastectomy. These authors had also compiled from the literature eight other cases of desmoid tumour in the thorax although they did not specify the exact site or details of these. Wiper and Miller (1946) and Carty (1954) have recorded other instances of desmoid tumours originating in the chest wall. 


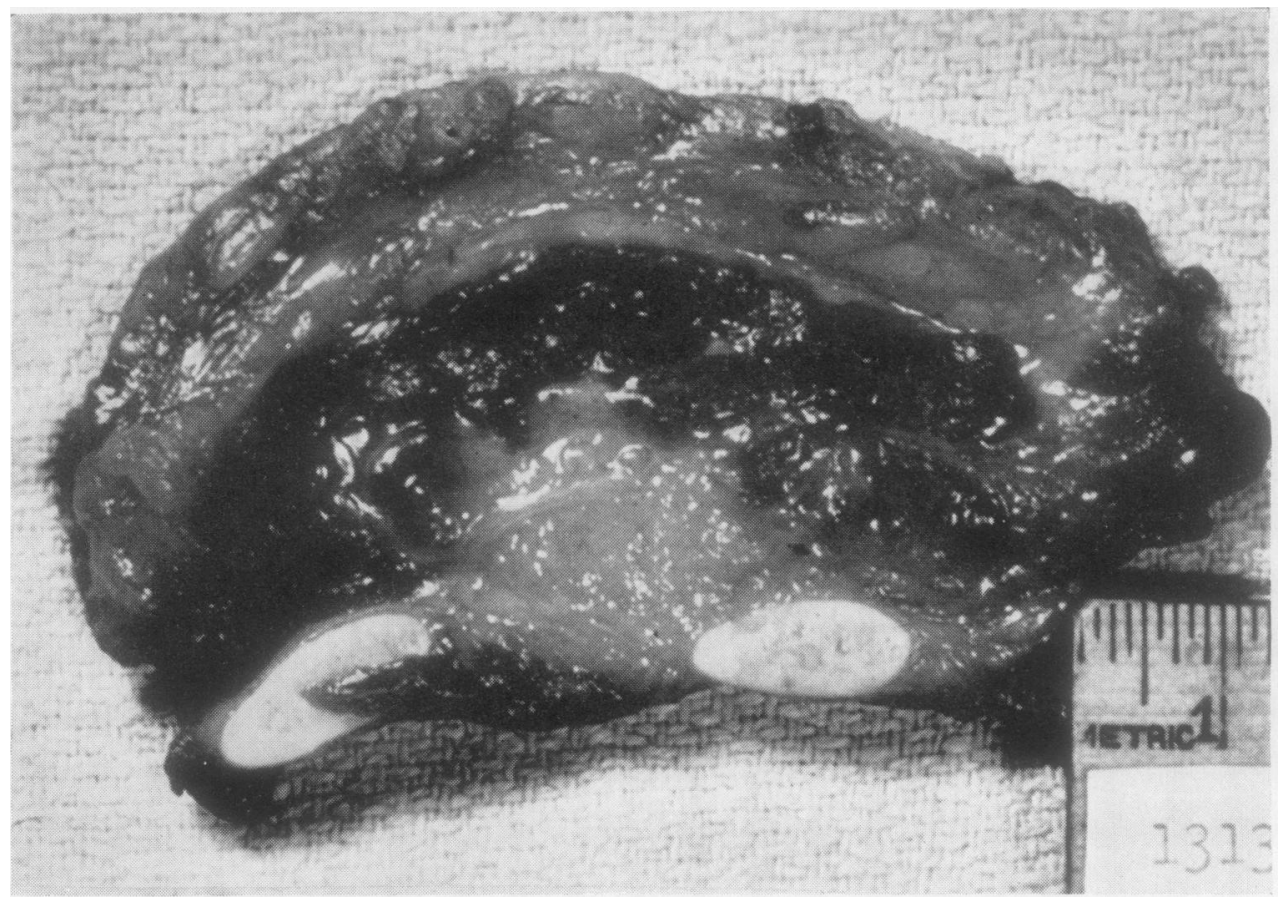

FIG. 2.-Gross specimen showing the tumour between the third and fourth rib cartilages, and adjacent chest wall structures. Note the lack of tumour encapsulation and diffuse invasion into contiguous muscles.

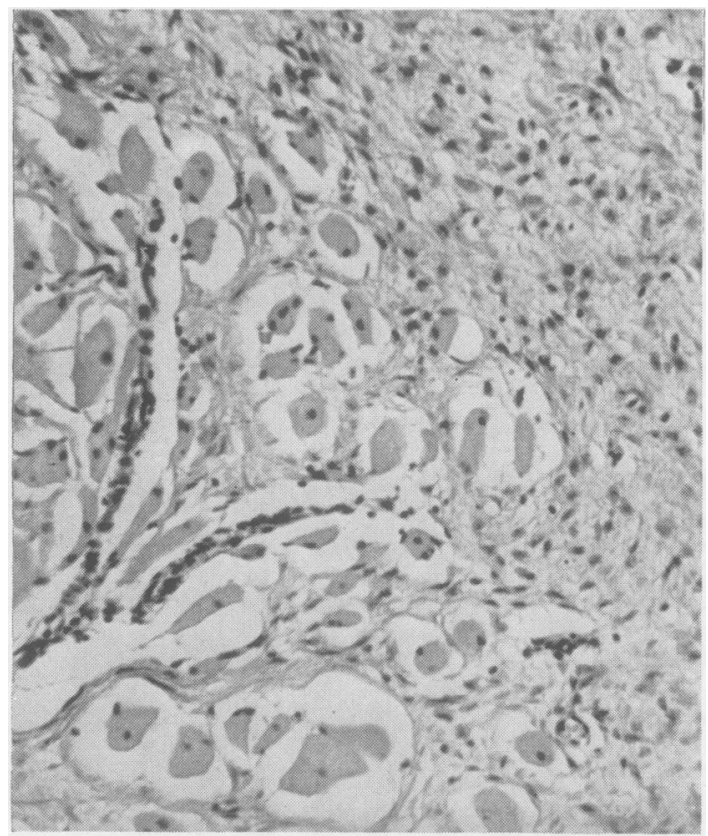

FIG. 3.-Illustrating the moderately cellular nature of this fibrous tumour, the microphotograph also shows invasion and infiltration of adjacent striated muscle.

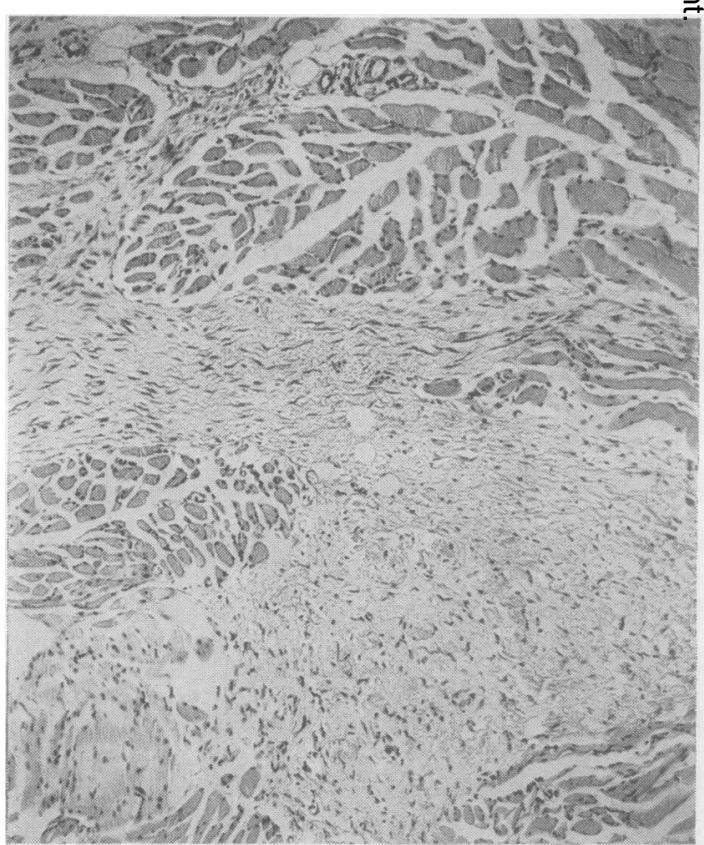

FIG. 4.-Lack of encapsulation and infiltration of muscle groups by the desmoid tumour is demonstrated by this photomicrograph. 
Baxter and Munro (1950) reviewed the published cases of fibroma of the chest wall.

Desmoid tumours must be distinguished histologically from fibrosarcomas, and because of their lack of encapsulation this may present difficulties on macroscopic examination.

Because these tumours and fibrosarcomas both infiltrate adjacent muscle, initial wide resection with a generous margin of normal tissue is mandatory. Local recurrences have been frequent when excision was inadequate. Incisional biopsy is contraindicated because of the possibility of disseminating malignant lesions that must be considered in the differential diagnosis of a desmoid.

There is little evidence in support that these tumours are radiosensitive, and accordingly the chief therapy must be surgical (Pack and Ehrlich, 1944). Although some authors have mentioned the endocrine relation of desmoids as shown by the high gonadotrophic assay of this tissue (Geschickter and Lewis, 1935), the use of antifibromatogenic hormones has not been adequately studied. Castration has appeared to benefit nonresectable tumours in isolated cases (Pack and Ehrlich, 1944). The occurrence of other fibromatous lesions in patients with desmoid tumours suggests the possibility of a systemic fibromatogenic principle. One of our patients with a desmoid in the anterior abdominal wall also had a fibromatous mass histologically resembling desmoid tissue in the mesentery at the site of a previous colectomy.

\section{SUMMARY}

A 21-year-old parous woman who had a firm tumour of the anterior chest wall presented herself for diagnosis and treatment. The chest wall in continuity with the tumour was widely excised. Histological examination of the specimen showed a desmoid tumour. No recurrence has been noted for 22 months post-operatively.

The literature regarding desmo:ds of the chest is briefly reviewed. Wide excision without incisional biopsy is advised for fibrous tumours of the thorax.

\section{REFERENCES}

Baxter, S. G., and Munro, D. D. (1950). J. thorac. Surg., 19, 944. Bennett, R. (1850). Lancet, 2, 328.

Carty, J. B. (1954). Amer. J. Surg., 87, 285.

Gatchell, F. G., Clagett, O. T., and McDonald, J. R. (1957). J. thorac Surg., 34, 184

Geschickter, C. F., and Lowis, D. (1935). Amer. J. Cancer, $25,630$.

Greig, D. M. (1915). Edinb. med. J., 15, 345.

MacFarlane, J. (1832). Clinical Reports of the Surgical Practice of the Glasgow Royal Infirmary, pp. 63 and 66. D. Robertson, Glasgow. Glasgow Royal Infirmary, pp. 63 and 66.
Mason, J. B. (1930). Ann. Surg., 92, 444.

Mason, J. B. (1930). Ann. Surg., 92, 444. haften Geschwülste. G. Reimer, Berlin.

Musgrove, J. E., and McDonald, J. R. (1948). Arch. Path. (Chicago), 45, 513.

Nichols, R. W. (1923). Arch. Surg. (Chicago), 7, 227.

Pack, G. T., and Ehrlich, H. E. (1944). Int. Abstr. Surg., 79, 177.

Pearman, R. O., and Mayo, C. W. (1942). Ann. Surg., 115, 114.

Stewart, M. J., and Mouat, T. B. (1924). Brit. J. Surg., 12, 355.

Waugh, J. M. (1940). Amer. J. Surg., 50, 694

Wiper, T. B., and Miller, J. M. (1946). Ibid., 71, 556. 